\title{
ESTIMATIVA DO BALANÇO DE ENERGIA PELO MÉTODO DA RAZÃO DE BOWEN A PARTIR DE MEDIDAS DE PROTÓTIPO DE ESTAÇÃO MICROMETEOROLÓGICA
}

\author{
SILVA FILHO, Armando da - armandosf2000@gmail.com \\ Universidade do Estado do Mato Grosso / UNEMAT
}

\author{
BIUDES, Marcelo Sacardi - marcelo@fisica.ufmt.br \\ Universidade Federal do Mato Grosso / UFMT
}

\author{
MACHADO, Nadja Gomes - nadja.machado@blv.ifmt.edu.br \\ Instituto Federal do Mato Grosso / IFMT
QUERINO, Carlos Alexandre Santos - carlosquerino@ufam.edu.br Universidade Federal do Amazonas / UFAM

\author{
ALMEIDA JÚNIOR, Elio Santos - elio_jr_@hotmail.com \\ Universidade Federal do Mato Grosso / UFMT
}

\begin{abstract}
RESUMO: O balanço de energia é de grande importância para a caracterização do microclima. Ele possibilita analisar a interação entre as variáveis ambientais e a superfície, bem como, identificar também efeitos decorrentes das atividades antrópicas. No entanto, estimar o balanço de energia em escala espacial pode ser inviável devido ao elevado custo de equipamento comercial. Sendo assim, o objetivo desse trabalho foi construir e avaliar o funcionamento de duas estações micrometeorológicas para estimar o balanço de energia pelo método de razão de Bowen em duas coberturas do solo distintas ao mesmo tempo. O estudo foi desenvolvido em uma área de cana-de-açúcar e numa área urbana residencial, distantes $25 \mathrm{~km}$, no município de Barra do Bugres-MT. Os saldos radiômetros, piranômetros, fluxímetros de calor no solo, termohigrômetros e datalogger foram desenvolvidos em laboratório e calibrados em campo. As medidas dos sensores desenvolvidos apresentaram alta correlação em relação aos sensores de referência. Devido à acurácia das medidas foi possível estimar os gradientes de temperatura e pressão de vapor d'água do ar. Assim, foi possível estimar o balanço de energia das duas áreas experimentais pelo método de Razão de Bowen a partir de medidas realizadas nos dois protótipos de estação micrometeorológica desenvolvidos.
\end{abstract}

PALAVRAS-CHAVE: Instrumentação micrometeorológica; Radiação solar; Desenvolvimento; Prototipação.

ENERGY BALANCE ESTIMATE BY BOWEN RATIO METHOD FROM MEASUREMENTS OF THE MICROMETEOROLOGICAL STATION PROTOTYPE

ABSTRACT: The energy balance is of great importance for the characterization of the microclimate. It allows to analyze the interaction between the environmental variables and the surface, as well as to identify also effects deriving from the anthropic activities. However, estimating the energy balance on a spatial scale may be impracticable due to the high cost of commercial equipment. Thus, the objective of this work was to construct and evaluate the operation of two micrometeorological stations to estimate the energy balance by the Bowen ratio method in two different soil coverages at the same time. The study was carried out in an area of sugarcane and a residential urban area, distant 25 $\mathrm{km}$, in the municipality of Barra do Bugres-MT. The net radiometers, pyranometers, soil heat flux meters, thermohygrometers and datalogger were developed in the laboratory and calibrated in the field. The measurements of the developed sensors presented a high correlation in relation to the reference sensors. Due to the accuracy of the measurements it was possible to estimate the temperature gradients and the water vapor pressure of the air. Thus, it was possible to estimate the energy balance of the two experimental 
areas by the Bowen Reason method from measurements performed on the two prototype micrometeorological station developed.

KEYWORDS: Micrometeorological instrumentation; Solar radiation; Development; Prototyping.

\section{INTRODUÇÃO}

O Cerrado mato-grossense tem-se destacado nos últimos anos pela alta capacidade de produção agrícola, em soja, milho, cana-de-açúcar e carne bovina (IBGE, 2014). Entretanto, estas atividades exigem a supressão do Cerrado alterando o albedo que é dependente do tipo de vegetação, coloração e rugosidade da superfície (SANO et al., 2010). Em consequência da alteração de um desses fatores, ocorre a alteração do saldo de radiação e, consequentemente, na temperatura da superfície. Essas alterações provocam modificações no balanço de energia superficial devido aos fluxos de calor latente e sensível (BIUDES et al., 2009).

O método da razão de Bowen (MRB) possibilita estimar a partição da energia disponível, em fluxos de calor latente e sensível, por meio de medidas realizadas diretamente no campo, como o saldo de radiação, fluxo de calor no solo e gradientes de temperatura e pressão de vapor d'água. O MRB tem sido amplamente utilizado desde sua proposição em 1926 por tem vantagens de ser de conceito físico, ter poucos parâmetros de entrada, e ser um método de cálculo simples (HU et al., 2013). A comparação dos fluxos de calor latente e sensível obtidos pelo MRB e método de covariância turbulenta mostram que, o MRB possibilita a estimativa de valores exatos e confiáveis, e que o desvio de leitura dos instrumentos é mínimo em um período inferior a um ano (DREXLER et al., 2004; BILLESBACH e ARKEBAUER, 2012; DICKEN et al., 2013).

Apesar do custo de aquisição e instalação do MRB ser menor que do método de covariância turbulenta, a estimativa do balanço de energia em escala espacial tem elevado custo. Isso ocorre, pois, os sensores e registradores de dados de uma estação micrometeorológica são importados. Dessa forma, a viabilização de uma estação micrometeorológica composta de sensores e componentes eletrônicos adquiridos no mercado nacional possibilitaria, o estabelecimento de uma rede de estações.

Alguns sensores micrometeorológicos têm sido desenvolvidos, como saldo radiômetros, piranômetros e fluxímetros de calor do solo e apresentam elevada sensibilidade e acurácia (NEVES, 2011; NEVES, 2013; NEVES et al., 2015). No entanto, esses sensores foram testados e validados individualmente com sensores comerciais e não foram utilizados em conjunto para estimar o balanço de energia no campo.

O desenvolvimento e teste a campo de estações micrometeorológicas possibilita consolidar a tecnologia de instrumentação já desenvolvida e minimiza a dependência de importação de equipamentos. Dessa forma, o objetivo desse trabalho foi construir e avaliar o funcionamento de duas estações micrometeorológicas para estimar o balanço de energia pelo método de razão de Bowen em duas coberturas do solo distintas ao mesmo tempo. 


\section{MATERIAL E MÉTODOS}

\section{1 ÁREA DE ESTUDO}

Os equipamentos desenvolvidos foram instalados em duas estações micrometeorológicas, instaladas em cobertura do solo distintas entre entre 01/10 e 03/10/2014, no município de Barra do Bugres-MT (Figura 1), distantes $25 \mathrm{~km}$. Uma delas estava localizada em um plantio de cana-de-açúcar na área rural (56 59' 33,486" W $15^{\circ} 8^{\prime} 2,829^{\prime}$ ' S e 205 m de altitude) e a outra numa

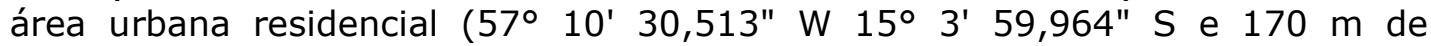
altitude). A estação micrometeorológica na área urbana foi instalada num quintal gramado. O dossel das edificações do entorno tem altura média de $4 \mathrm{~m}$ e possui calçadas de concreto e ruas de asfalto.

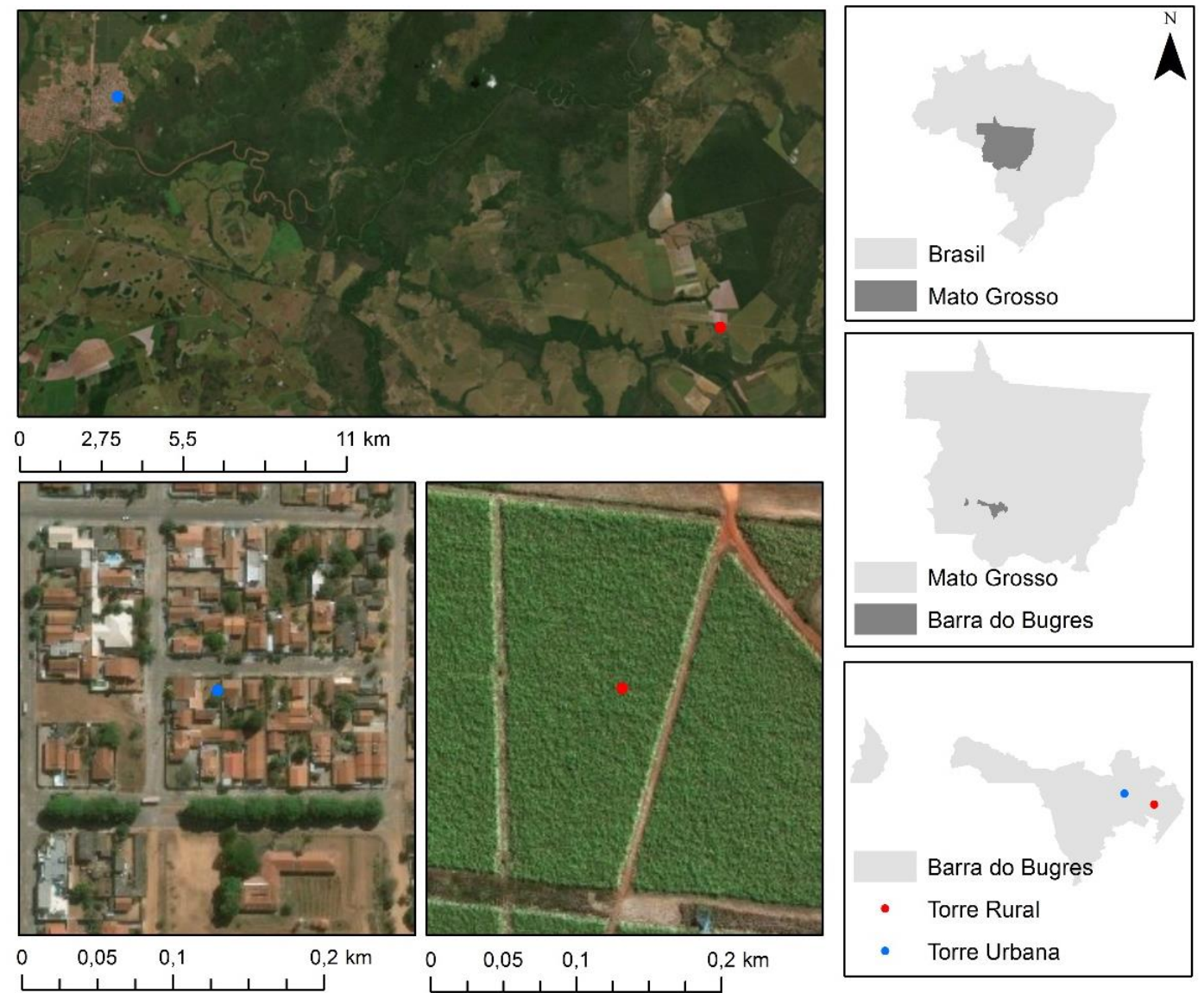

Figura 1- Localização das torres micrometeorológicas desenvolvidas.

\subsection{PIRANÔMETRO}

O piranômetro foi desenvolvido de acordo com as especificações propostas por Neves $(2011 ;$ 2013). O corpo do piranômetro desenvolvido consistiu de um cilindro sólido de alumínio usinado, com três níveis de rebaixo consecutivos (Figura 2a). O primeiro rebaixo foi usado para acomodação de uma placa de circuito impresso em forma de disco como suporte do sensor. O segundo rebaixo foi usado para acomodação do sensor (BPW34, Vishay 
Semiconductors) (Figura $2 \mathrm{~b}$ ). O terceiro rebaixo (Figura $2 \mathrm{c}$ ) foi utilizado para encaixe do colimador de acrílico (Figura 2d). A parte traseira da placa com o sensor foi usada de base para a resistência de shunt (Figura $2 \mathrm{e}$ ). O cabo de sinal do sensor foi acomodado em um furo na lateral do corpo do piranômetro (Figura 2f). A base do piranômetro (Figura $2 \mathrm{~g}$ ) também foi construída em alumínio torneado para encaixe na parte inferior do corpo do piranômetro. Após a montagem do piranômetro, a parte interna foi selada com borracha de silicone para evitar entrada de umidade (Figura $2 \mathrm{~h}$ ).
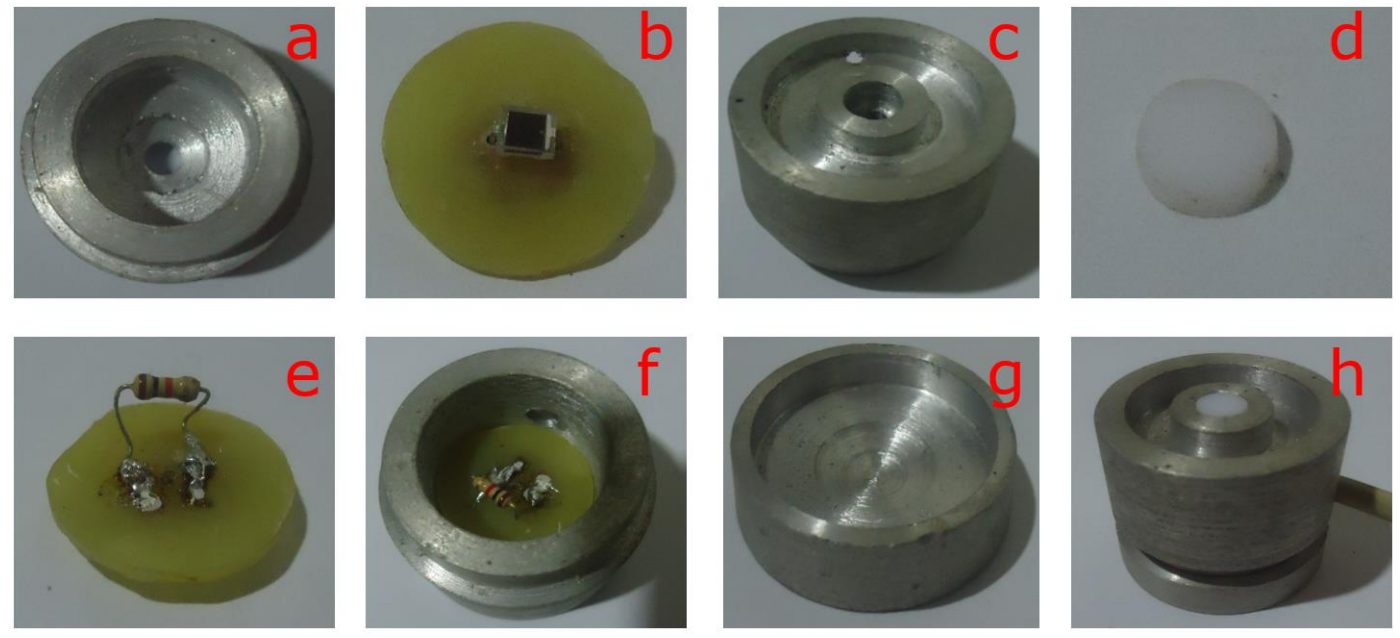

Figura 2 - Etapas de construção dos piranômetros desenvolvidos.

\subsection{SALDO RADIÔMETRO E FLUXÍMETRO DE CALOR NO SOLO}

O saldo radiômetro e o fluxímetro de calor no solo foram construídos de maneira semelhante, utilizando duas (2) pastilhas Peltier (TEC-112706, Acel) sobrepostas (Figura 3a). As duas placas de Peltier foram unidas com pasta térmica e foram ligadas eletricamente em série, proporcionado uma maior diferença de potencial pela mesma diferença de temperatura entre as faces superior e inferior. O conjunto foi inserido em uma forma cilíndrica de acrílico (Figura 3b) e envolvida em resina de poliéster (Figura 3c), mantendo uma camada de $0.5 \mathrm{~mm}$ de resina entre a face superior e inferior, com uma haste de fixação na lateral para acomodar os cabos. Após a resina de poliéster secar, o saldo radiômetro foi pintado de cor preta (Figura 3d) para facilitar o aquecimento da superfície, já que a resina tem cor clara.

O fluxímetro de calor do solo foi construído sem a haste de fixação e foi pintada de alaranjado, para facilitar a instalação no solo e identificação do lado superior. A Figura 3e mostra o fluxímetro de calor no solo pronto para receber resina de poliéster. Na sequência, o sensor já em processo de cura e desmoldado antes do acabamento (Figuras $3 f$ e $3 g$ ) e após a pintura (Figura 3h). 

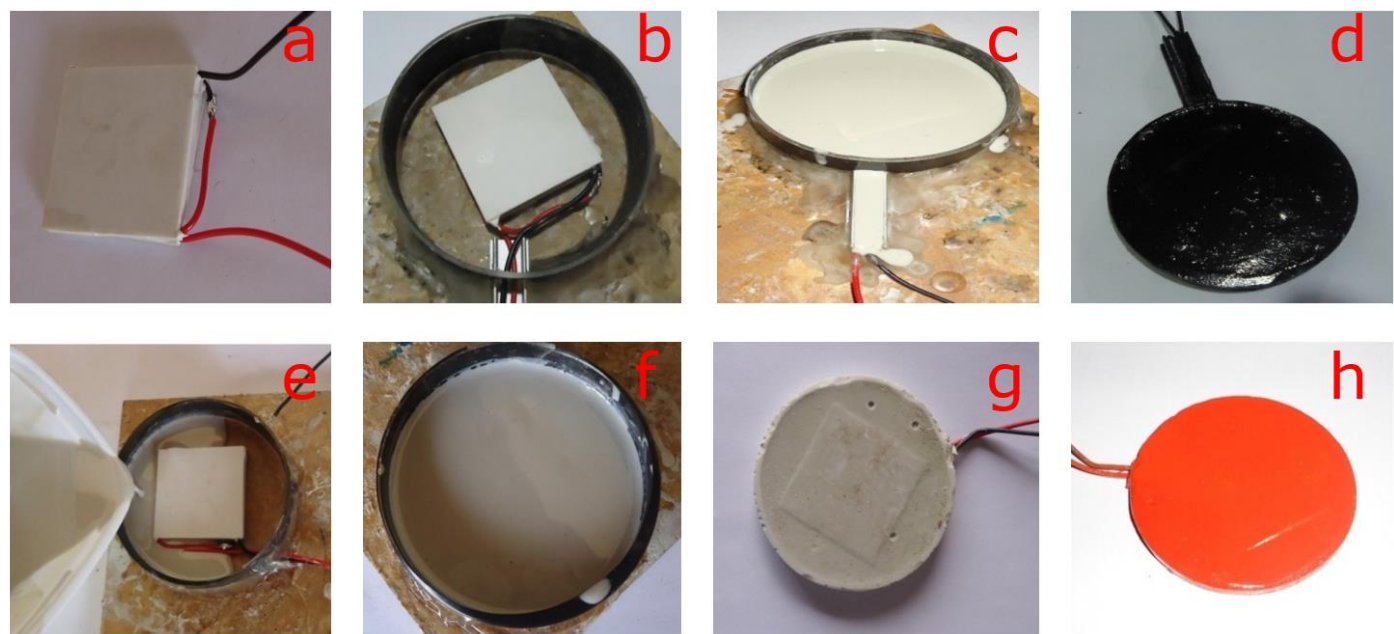

Figura 3 - Etapas de construção do saldo radiômetros (a-d) e dos fluxímetros de calor no solo (e-h) desenvolvidos.

\subsection{TERMOHIGRÔMETRO E ABRIGO}

O sensor termohigrômetro usado neste experimento foi o modelo DHT22, por englobar características desejáveis como precisão, baixo consumo, comunicação digital, resistência a intempéries e calibração e certificação de fábrica (Figura 4). Este sensor possui um sensor de umidade capacitivo e um sensor de temperatura NTC, conectado a um microcontrolador de 8 bits responsável pela leitura dos sensores e conversão dos dados analógicos em digitais, com capacidade de comunicação de até 20 metros.

O conjunto sensor foi protegido por um abrigo meteorológico de PVC branco, para evitar a exposição direta dos sensores aos raios solares e a chuva. Cada abrigo foi construído com 3 suportes de PVC sobrepostos (Figura 4a) usados em iluminaria, e o topo coberto com um nipel de PVC (Figura 4b); 0 conjunto foi fixado por 2 parafusos nas laterais com espaçadores de $10 \mathrm{~mm}$ entre cada suporte (Figura 4c). Dessa forma, garantiu-se a livre circulação do ar e permitiu o equilíbrio térmico e higrométrico do sensor e da atmosfera.
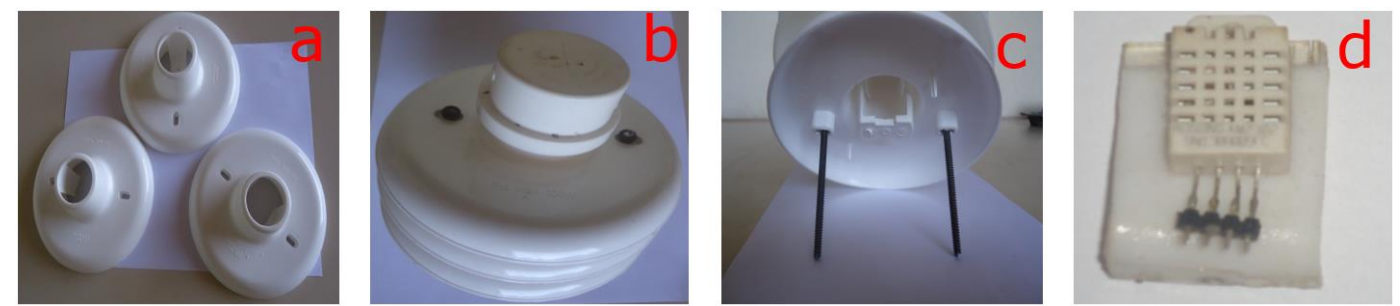

Figura 4 - Etapas de construção do abrigo termométrico e fixação do termohigrômetro usado nas estações micrometeorológicas.

\subsection{REGISTRADOR DE DADOS}

O registrador de dados (datalogger), foi desenvolvido para atender as seguintes especificações: (I) coleta de dados via pen drive para facilitar a coleta dos dados no campo, sem a necessidade de computadores ou outro recurso 
computacional, aliada a alta capacidade de armazenamento de dados; (II) gravação dos dados no formato texto (*.txt), o qual é padrão de softwares de planilhas eletrônicas e banco de dados; (III) possibilidade de reprogramação em campo, quando da adição ou retirada de sensores; (IV) baixo consumo de energia; (V) elevada autonomia; (VI) dezesseis entradas analógicas e três entradas digitais; e (VII) sinalizador visual do status do equipamento.

Para isso foi utilizado o microcontrolador (18f452, Microchip Technology Inc) que apresenta $32 \mathrm{~kb}$ de memória, conversor $A / D$ (analógico/digital) de 10 bits, 33 pinos de entrada e/ou saída disponíveis e baixo consumo de energia. A sincronia temporal foi obtida do relógio de tempo real (ds1302, Maxim Integrated) por possuir comunicação serial bidirecional compatível com os níveis de tensão do microcontrolador utilizado, baixo consumo de energia e sistema de bateria auxiliar. A necessidade de entradas analógicas em número superior às disponíveis no microcontrolador exigiu o uso de 2 multiplexadores analógicos (CD4051 Texas Instruments).

O armazenamento dos dados em memória flash (pen drive) foi feito com um módulo embarcado (PenBS Tato Equipamentos Eletrônicos Ltda), por possuir comunicação serial assíncrona compatíveis com microcontroladores e a gravação dos dados diretamente em pen drive no formato de sistema de arquivos FAT 32 . Assim, foi possível coletar os dados apenas com uma simples troca de pen drive.

Visando maior flexibilidade do datalogger, todo o projeto foi dividido nos módulos: (I) uma placa mãe (Figuras 5a e 5b); (II) multiplexador ADC (Figura 5c); (III) relógio de tempo real (Figura 5d); (IV) painel de bornes (Figura 5e), o que permite facilidade do projeto lógico, testes em simuladores, desenvolvimento da placa de circuito impresso, montagem das placas, testes e aferição individual dos módulos antes da montagem final do datalogger (Figura $5 f)$.
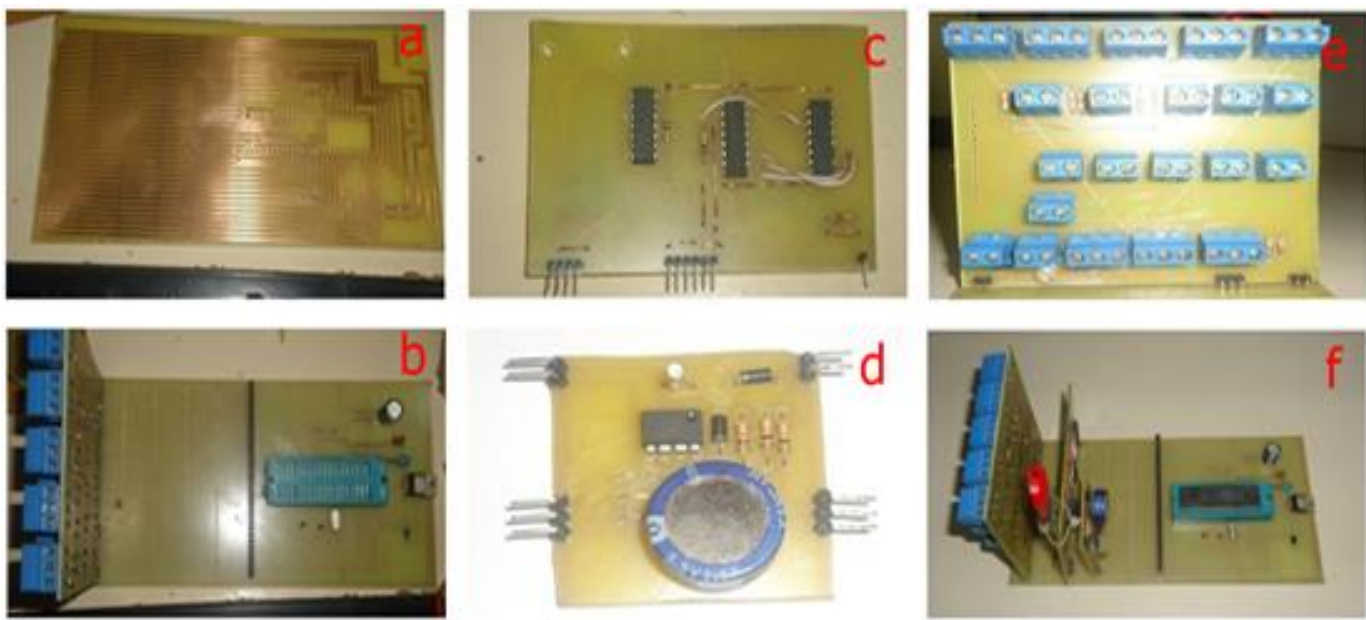

Figura 5 - Módulos do registrador de dados das estações micrometeorológicas.

O sistema foi alimentado por uma bateria de $12 \mathrm{~V}$ e $7 \mathrm{Ah}$, mantida em flutuação com um painel solar de $5 \mathrm{~W}$. O microcontrolador foi programado para leitura de todas as entradas a cada 2 segundos e a gravação das médias a cada minuto para maior granularidade de dados. 
Leds luminosos foram instalados no painel de bornes para indicar o status de funcionamento de todo o equipamento: (I) pulsos rápidos indicavam que as leituras estavam sincronizando com relógio de tempo real; (II) pulsos intermitentes indicavam que estava em processo de leitura de dados; (III) led aceso sem intermitência indicava que estava em processo de gravação dos dados; (IV) e led apagado indicava defeito ou desligado da alimentação elétrica.

\subsection{CALIBRAÇÃO DOS INSTRUMENTOS DESENVOLVIDOS}

A calibração de cada sensor foi feita por regressões lineares simples, adotando como referência os sensores comerciais equivalentes. Os sensores de radiação e fluxo de calor no solo foram instalados ao lado de sensores comerciais para conversão dos sinais em $\mathrm{mV}$ para $\mathrm{W} \mathrm{m}-2$. Para isso, foram utilizados um piranômetro (LI-200, Campbell Sci, Inc., USA), um sensor de fluxo de calor no solo (HFP01-15, Thermal Sensors, Hukseflux) e um saldo radiômetro (NR-LITE25, Kipp e Zonen Netherlands). Esses sensores foram conectados a um datalogger (CR 10X, Campbell Scientific, Inc., Logan, Utah).

Cada sensor DHT22 utilizado para construção de cada termohigrômetro possui certificação do fabricante. Não necessitando de uma calibração com sensores comerciais. No entanto, para retirar um possível efeito da estrutura do abrigo meteorológico fabricado na obtenção do gradiente de temperatura e pressão de vapor do ar entre cada par de termohigrômetro, foram estabelecidas relações entre a temperatura, umidade relativa e pressão de vapor do ar entre cada par de conjunto de termohigrômetro (abrigo e sensor).

Todos os sensores foram dispostos sobre um mesmo gramado homogêneo para serem calibrados simultaneamente sob as mesmas condições meteorológicas em relação aos sensores comerciais certificados. As medidas para calibração foram realizadas durante 1 semana para avaliar a eficiência do painel solar, das baterias, realizar testes de possíveis interferências nas leituras devido temperatura interna no invólucro do datalogger e resistência do circuito à umidade atmosférica.

\subsection{DISPOSIÇÃO DOS SENSORES}

Após todos os sensores desenvolvidos serem calibrados, todos foram afixados em uma haste de aço. O saldo de radiação e a radiação solar incidente e refletida foi medido a $3 \mathrm{~m}$ de altura do solo na área de cana-de-açúcar $(1 \mathrm{~m}$ acima do dossel) e a $6 \mathrm{~m}$ de altura do solo na área urbana ( $2 \mathrm{~m}$ acima do dossel médio das casas). O fluxo de calor no solo foi medido a $2 \mathrm{~cm}$ de profundidade do solo nas duas áreas experimentais. O perfil de temperatura e umidade relativa do ar foi estimado a partir de 2 alturas nas duas áreas experimentais: a $3 \mathrm{~m}$ e $6 \mathrm{~m}$ de altura do solo na área de cana-de-açúcar e $6 \mathrm{~m}$ e $9 \mathrm{~m}$ de altura do solo na área urbana.

\subsection{ESTIMATIVA DO BALANÇO DE ENERGIA}

Os fluxos de calor latente (LE) e sensível $(H)$ foram estimados pelo MRB. Os fluxos médios de LE (Equação 1$)$ e H (Equação 2) $\left(\mathrm{W} \mathrm{m}^{-2}\right)$ foram calculados, em intervalos de 30 minutos. 
$L E=\frac{R n-G}{1+\beta}$

$H=\frac{R n-G}{1+\beta^{-1}}$

em que, Rn é o saldo de radiação $\left(\mathrm{W} \mathrm{m}^{-2}\right)$, G é o fluxo de calor no solo $\left(\mathrm{W} \mathrm{m}^{-2}\right)$ e $\beta$ é a razão de Bowen (Equação 3):

$\beta=\left(\frac{C_{p}}{\lambda 0.622}\right)\left(\frac{\Delta T}{\Delta e}\right)$

em que, $C_{p}$ é o calor específico em pressão constante $\left(1.00467 \mathrm{~J} \mathrm{~g}^{-1} \mathrm{~K}^{-1}\right)$, $\lambda$ é o calor latente de vaporização $\left(\mathrm{J} \mathrm{g}^{-1}\right)$ definido pela Equação (4), 0.622 é a razão do peso molecular da água e do ar, e $\Delta T$ e $\Delta e$ são as diferenças de temperatura $\left({ }^{\circ} \mathrm{C}\right)$ e pressão de vapor de água $(\mathrm{kPa})$, respectivamente, entre as duas diferentes alturas de medidas.

$\lambda=1.919 \times 10^{6}\left(\frac{T+273.16}{(T+273.16)-33.91}\right)^{2}$

O critério para aceitar e/ou rejeitar os dados obtidos pelo MRB foram baseados naqueles descritos por Perez et al. (1999). Assumiu-se que os gradientes de temperatura e pressão entre os termohigrômetros foram suficientemente distantes. O MRB falha quando (1) a resolução do sensor é inadequada para resolver os gradientes em $\Delta T$ e $\Delta e$ (UNLAND et al., 1996), (2) condições atmosféricas estáveis causam $\beta \approx-1$, como durante ao amanhecer e ao anoitecer (ORTEGA-FARIAS et al., 1996), e (3) as condições mudam abruptamente levando à erros de medição (PEREZ et al., 1999). Usando este método de filtragem, valores fisicamente realistas de $\beta$ podem ser obtidos de forma objetiva e quantitativa que limita o potencial de viés e erro na estimativa em termos do balanço de energia (PEREZ et al., 1999).

\section{RESULTADOS E DISCUSSÃO}

\subsection{CALIBRAÇÃO DOS SENSORES DESENVOLVIDOS}

Todos os sensores desenvolvidos, apresentaram uma alta correlação e significância (valor $P<0,001$ ) com os sensores de referência (Figuras 6 e 7). 

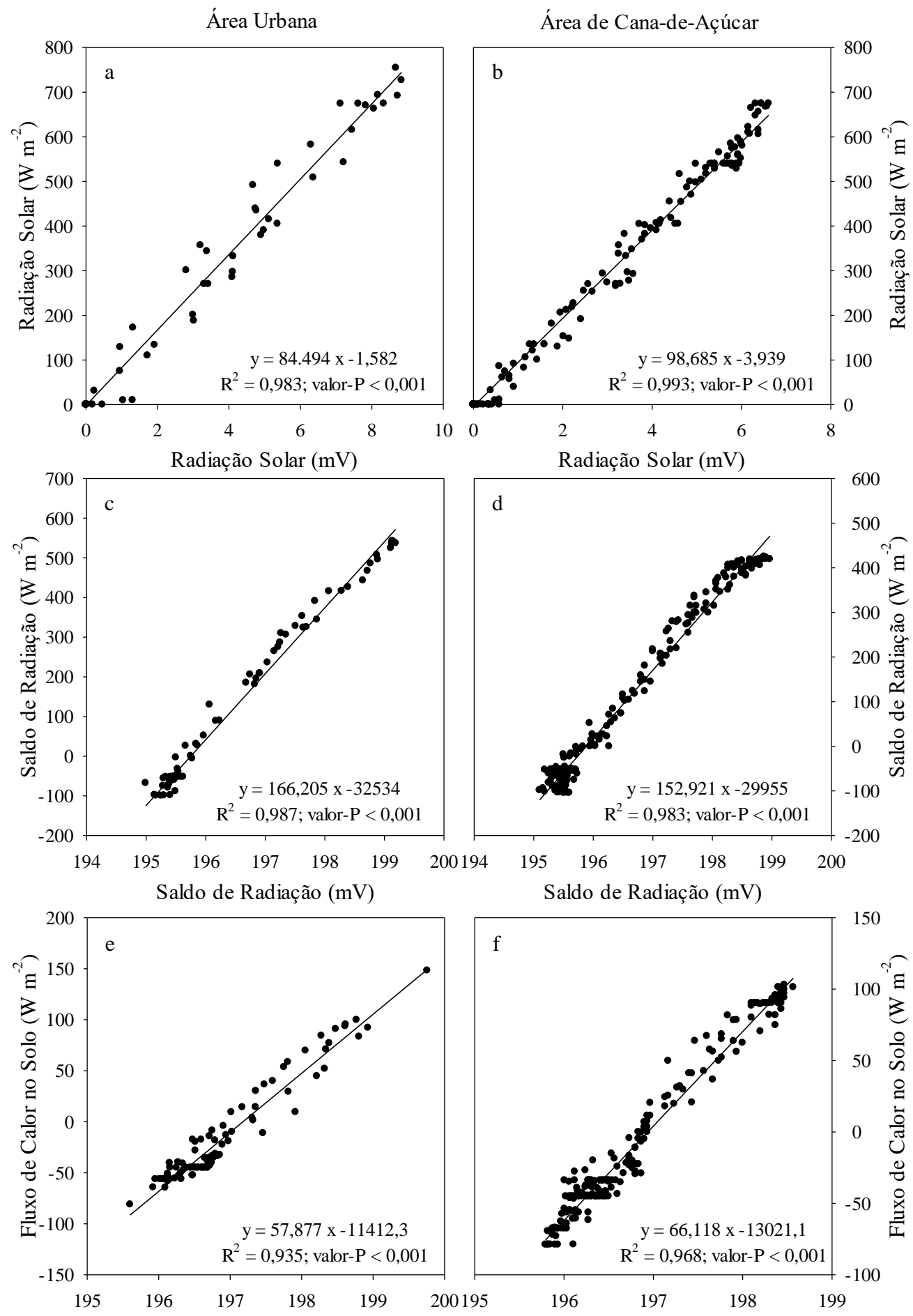

Fluxo de Calor no Solo (mV)

Fluxo de Calor no Solo (mV)

Figura 6 - Calibração (a e b) dos piranômetros, (c e d) dos saldos radiômetros, (e e f) dos fluxímetros de calor do solo desenvolvidos nesse trabalho (eixo $\mathrm{x}$ ), tendo como referência sensores comerciais (eixo y), que foram usados nas áreas urbana e de canade-açúcar, respectivamente, no município de Barra do Bugres-MT. 

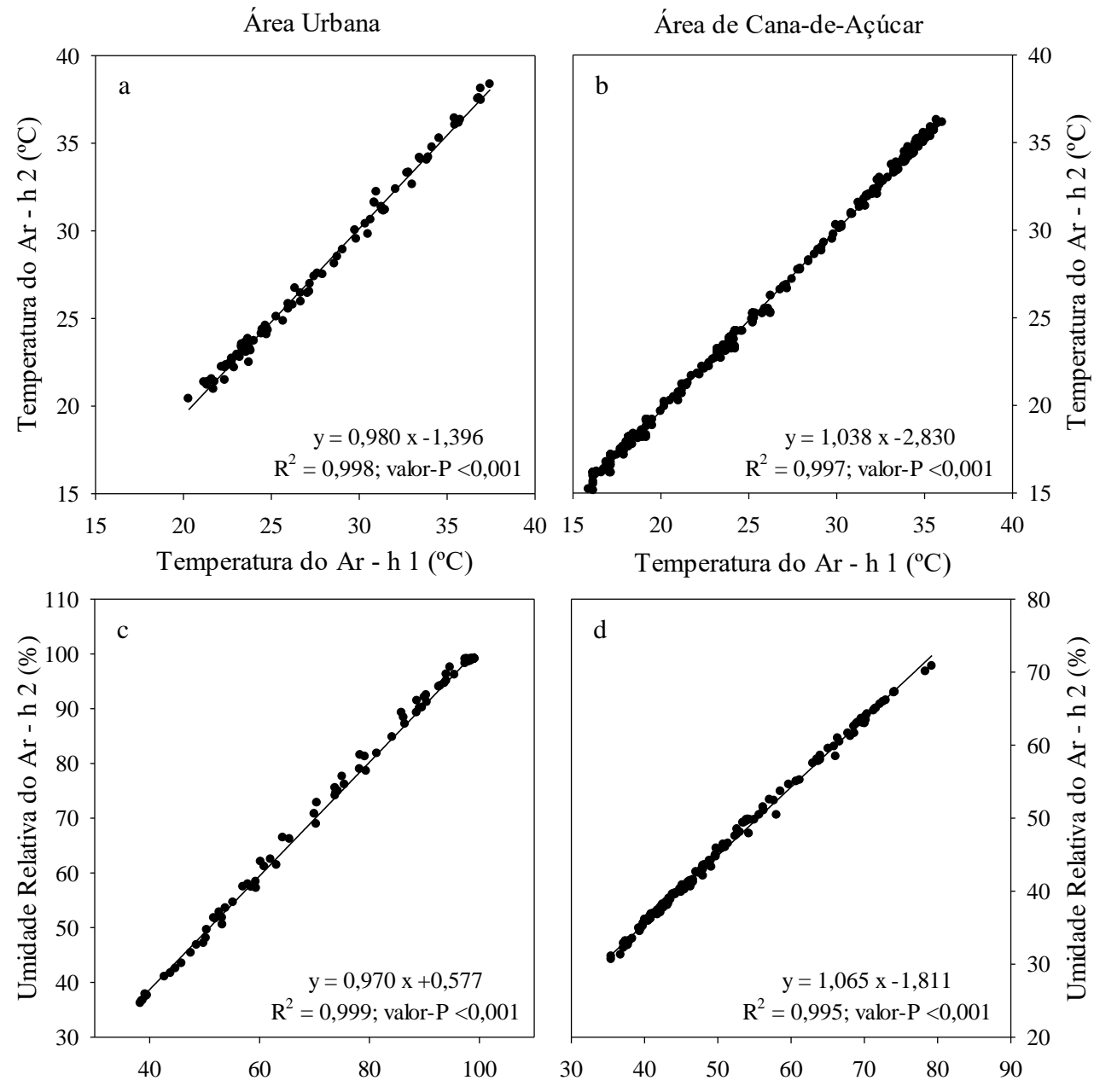

Umidade Relativa do $\mathrm{Ar}-\mathrm{h} 1(\%)$
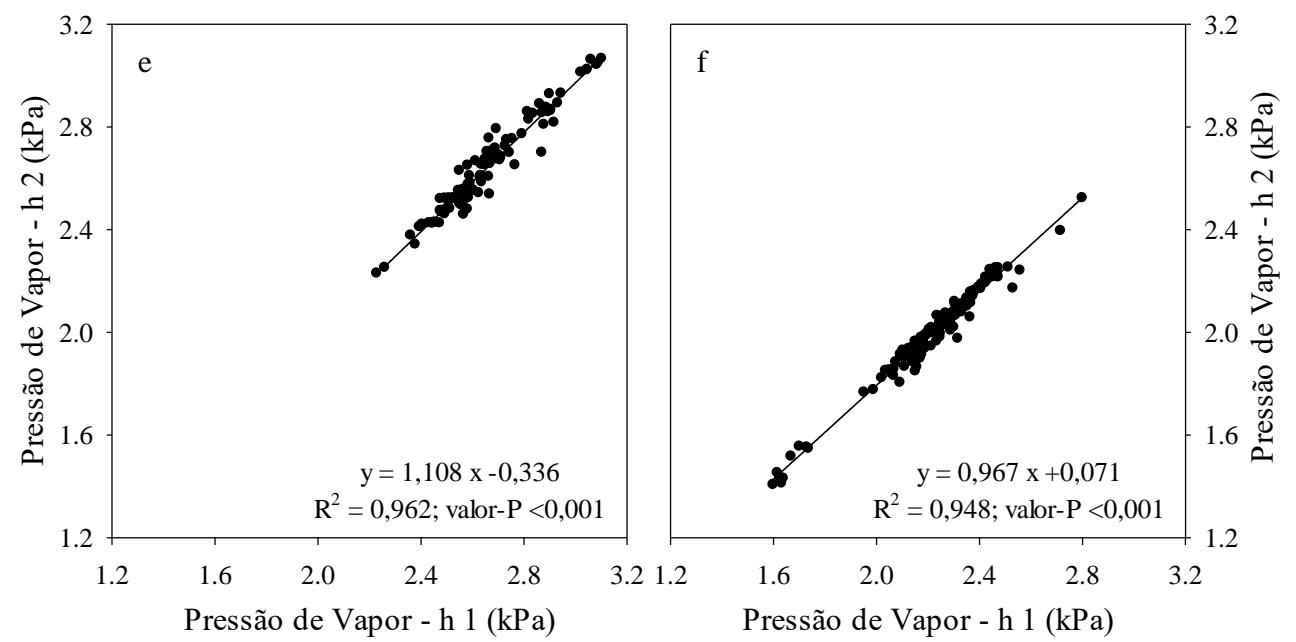

Figura 7 - Calibração (a e b) da temperatura do ar, (c e d) da umidade relativa do ar, (e e f) da pressão de vapor d'água dos termohigrômetros desenvolvidos nesse trabalho (eixo $x$ ), tendo como referência um termohigrômetro comercial (eixo y), que foram usados nas áreas urbana e de cana-de-açúcar, respectivamente, no município de Barra do Bugres-MT. 


\subsection{ANÁLISE DAS MEDIDAS COM OS SENSORES DESENVOLVIDOS}

A radiação solar incidente $(\mathrm{Rg})$ nos dois locais ocorreram entre 06:30 e 17:00 horas, com valor máximo de aproximadamente $800 \mathrm{~W} / \mathrm{m} 2$ às 12:00 horas (Figura 8a). O padrão da $\mathrm{Rg}$ nos dois locais foi semelhante com pequena variação, provavelmente devido a nebulosidade, já que as duas áreas experimentais estavam a $25 \mathrm{~km}$ uma da outra.

A temperatura do ar na área urbana teve mínimas e máximas diárias ligeiramente superiores às da área de cana-de-açúcar (Figura 8b). A temperatura do ar mínima ocorreu às 06:30 horas, quando aumentou devido ao aumento da $\mathrm{Rg}$, e atingiu máximo entre 12:00 e 15:00 horas. No dia 01/10/2014, observou-se um rápido declínio da temperatura horária, entre 14:00 e 16:00 horas, com queda acentuada de $3^{\circ} \mathrm{C}$ em duas horas, coincidindo com redução da $\mathrm{Rg}$ e aumento brusco da umidade relativa do ar (Figura 8c). A diminuição da $\mathrm{Rg}$ e da temperatura do ar e aumento da umidade relativa do ar nesse dia foi devido a um evento de chuva em ambas áreas experimentais. $\mathrm{Na}$ tarde do dia 01/10/2014, foi observado um total pluviométrico de $3,2 \mathrm{~mm}$ na área com cana-de-açúcar e 7,1 $\mathrm{mm}$ na área urbana (dos quais 5,2 mm ocorreram entre 14 e 16 horas). Em outubro inicia o período chuvoso na região. As temperaturas elevadas e o aumento de umidade durante a manhã favorecem a convecção e por consequência a formação de tempestades, o que favorece a precipitação no período vespertino (QUERINO et al., 2017). A queda da temperatura do ar no período noturno ocorreu lentamente mantendo o mesmo padrão em ambos os sítios.

A umidade relativa do ar nos dois sítios também teve padrão semelhante no período diurno (Figura 8c). A marcha diária da umidade relativa do ar inicia sua queda com o início da $\mathrm{Rg}$ e tem o mínimo diário coincidente com o máximo de temperatura. A umidade relativa do ar na área de cana-de-açúcar foi maior no período noturno durante todos os dias estudados, atingindo máximas de $100 \%$ quando a temperatura do ar esteve abaixo de $25^{\circ} \mathrm{C}$.

As diferenças nos elementos meteorológicos entre os dois sítios estão relacionadas à diferença na quantidade de vegetação no entorno. O entorno da área de cana-de-açúcar é toda vegetada, enquanto que na área urbana tem menos vegetação, e mais materiais predominantes como calçadas, asfalto e concreto das edificações. O material da área urbana armazena e conduz mais rapidamente $\mathrm{o}$ calor no período diurno que superfícies vegetadas (ALVES e VECCHIA, 2012). Esse padrão também foi observado por Callejas (2012) quando avaliou diferentes tipos de ambientes urbanos na cidade de Cuiabá-MT. 


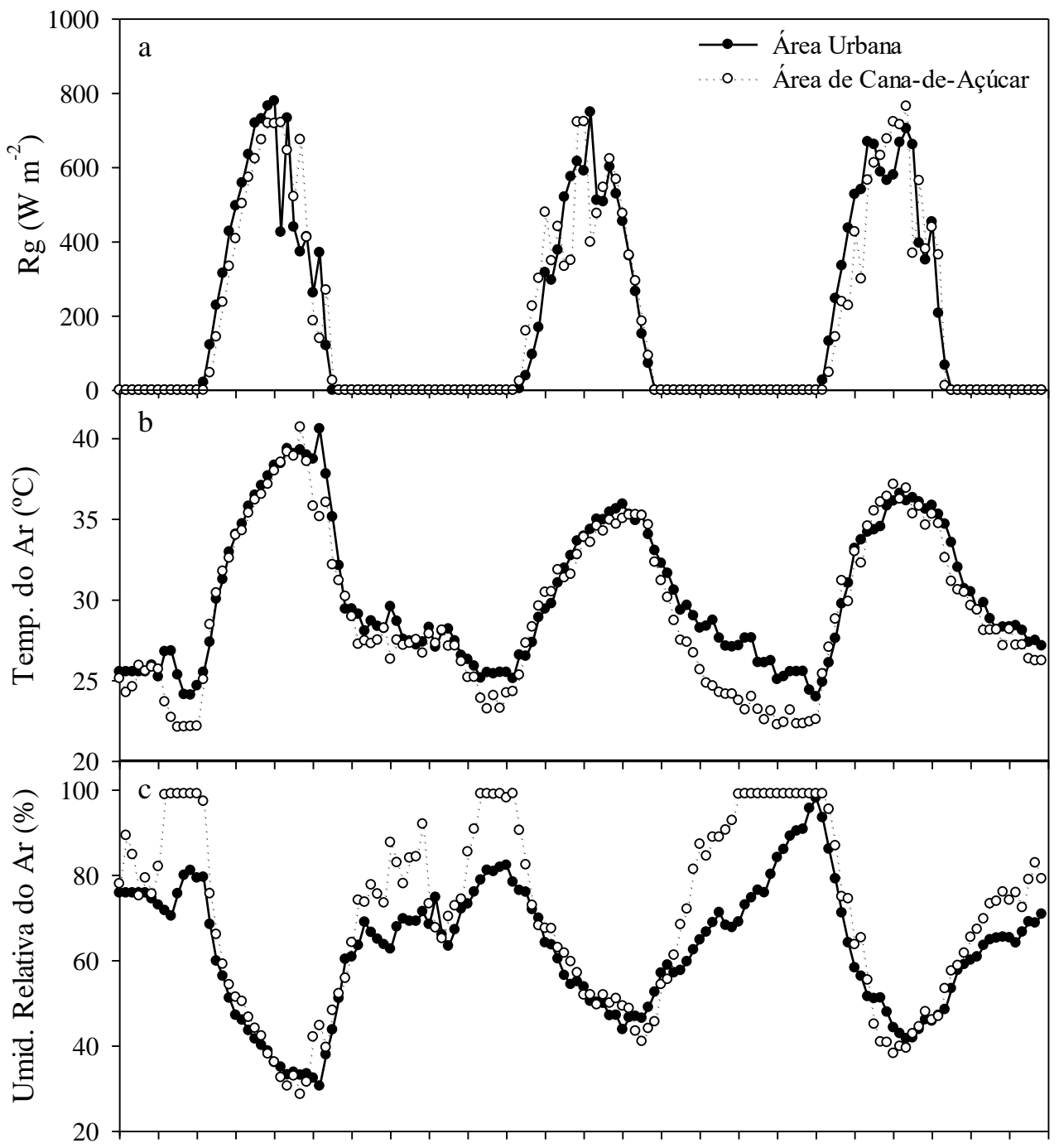

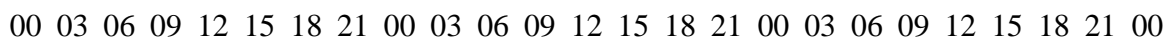
$01 / 10 / 2014$

$02 / 10 / 2014$

$03 / 10 / 2014$

Hora e Dia

Figura 8 - Variação horária da (a) radiação solar incidente, (b) temperatura do ar e (c) umidade relativa do ar medidas na área urbana e de cana-de-açúcar no município de Barra do Bugres-MT.

\subsection{ANÁlise do bALANÇO de enERgia PELO MÉtodo dA RAZÃo de BOWEN}

O saldo de radiação $(\mathrm{Rn})$ seguiu o padrão da $\mathrm{Rg}$ com valores positivos entre 06:30 e 17:00 horas, com valores máximos às 12:00 horas, e negativos durante o período noturno (Figura 9a). Os valores negativos do Rn noturno nos dois sítios foram devido à radiação de onda longa proveniente da superfície ser maior que a provenientes da atmosfera, e ausência completa do balanço de ondas curtas, o qual tende a dominar o balanço de radiação diurno (Querino et al., 2017). 
As diferenças no $\mathrm{Rn}$ entre as áreas foram devido às características térmicas e espectrais, influenciadas pelo albedo da superfície, geometria da área urbana, propriedades térmicas superficiais e disponibilidade de água no solo (CHRISTEN e VOGT, 2004; COUTTS et al., 2007; CHAKRABORTY et al., 2016).

Os valores mais negativos do Rn durante o período noturno ocorreram na área de cana-de-açúcar devido à maior quantidade de vegetação. A superfície vegetada possui maior quantidade de água que superfícies impermeabilizadas como asfalto e concreto, e por conseguinte, maior calor específico. Dessa forma, a vegetação atua como termorregulador da superfície, i.e., a temperatura da superfície na área vegetada é menor durante o período diurno e maior durante o período noturno em relação à área urbana (CHAKRABORTY et al., 2016). Portanto, a superfície da área de cana-de-açúcar emite mais radiação de onda longa no período noturno que na área urbana.

O fluxo de calor no solo (G) foi menor na área de cana-de-açúcar, representando em média de $1 \%$ do $\mathrm{Rn}$ ao longo dos três dias, enquanto que na área urbana representou $31 \%$ do Rn (Figura 9b). O G foi negativo durante 0 período noturno, sendo positivo a partir das 09:00 horas com máximo às 15:00 horas, indicando que a superfície transmite energia para as camadas mais profundas do solo, voltando a ser negativo após às 16 horas, quando o solo começa a perder calor para a atmosfera novamente.

Os menores valores do $G$ para a área de cana-de-açúcar foram devido à maior atenuação da $\mathrm{Rg}$ pela vegetação. Dessa forma, o $\mathrm{G}$ na área de cana-deaçúcar teve valores muito próximos de zero durante a maior parte do dia com máximo de $45 \mathrm{~W}$ m-2 em céu claro. Os valores de $\mathrm{G}$ nesse trabalho foram bem próximos dos $58 \mathrm{~W}$ m-2 encontrados por Romisio et al. (2010) e Ferreira et al. (2013) em um cultivo da cana-de-açúcar no mesmo estágio fenológico que se encontrava a cultura neste trabalho. Quanto à comparação entre área urbana e de cana-de-açúcar vegetada, outros autores também encontraram valores semelhantes a este trabalho, como Souza et al. (2012) em Ourinhos-SP, e Andrade et al. (2007) em Petrolina-PE, Juazeiro-BA e Juiz de Fora-MG, os quais compararam o microclima urbano com de áreas vegetadas.

O padrão horário do fluxo de calor latente (LE) nos dois sítios (Figura 9c) foram semelhantes ao do Rn, porém com intensidade diferente. O LE da área de cana-de-açúcar foi maior que na área urbana, e representou em média de $58 \%$ do $\mathrm{Rn}$, enquanto que na área urbana o LE representou $44 \%$ do $\mathrm{Rn}$. A diferença no LE entre os sítios foi função da maior quantidade de vegetação e maior disponibilidade hídrica na área de cana-de-açúcar. O fluxo de calor sensível $(H)$ nos dois sítios também teve padrão semelhante ao Rn (Figura 9d). No entanto, ao contrário do que se esperava, o fluxo de calor sensível foi maior na área de cana-de-açúcar, representando em média de $41 \%$ e $26 \%$ do $\mathrm{Rn}$ na área de cana-de-açúcar e urbana respectivamente. Apesar de ter menor $\mathrm{H}$ na área urbana, a maior parte do $\mathrm{Rn}$ nesse sítio foi utilizada para aquecimento da superfície (56\%), i.e., na forma de calor sensível para aquecimento do ar e do solo (Figura 10). A superfície vegetada normalmente utiliza maior parte da energia disponível para o processo de evapotranspiração (BIUDES et al., 2009; BIUDES et al., 2015), enquanto que a superfície quase toda impermeabilizada, como na área urbana, utiliza a maior parte da energia disponível para o processo de aquecimento da superfície (CALLEJAS et al., 2016). 


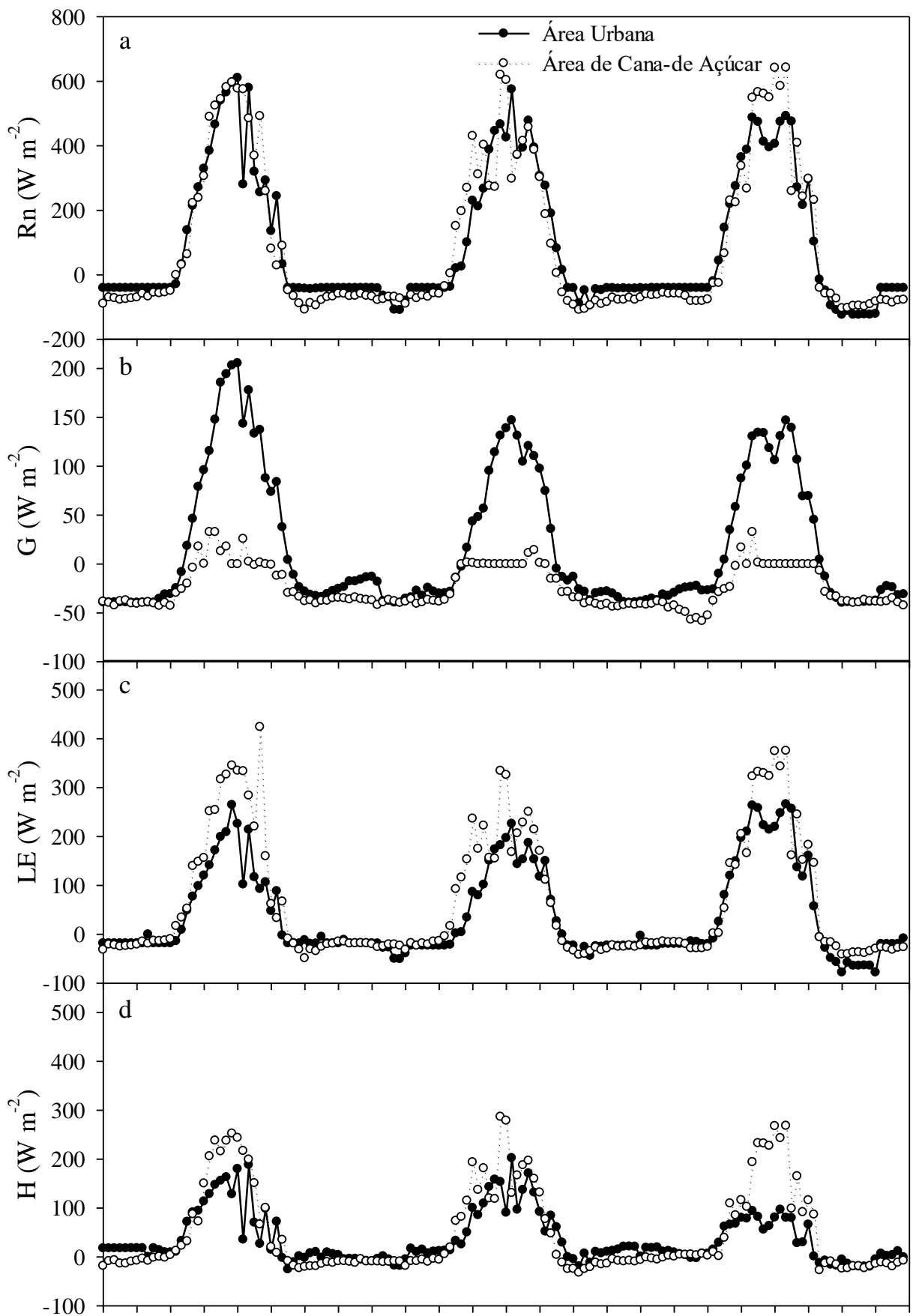

$\begin{array}{lllllllllllllllllllllllll}00 & 03 & 06 & 09 & 12 & 15 & 18 & 21 & 00 & 03 & 06 & 09 & 12 & 15 & 18 & 21 & 00 & 03 & 06 & 09 & 12 & 15 & 18 & 21 & 00\end{array}$ $01 / 10 / 2014$

$02 / 10 / 2014$

$03 / 10 / 2014$

Hora e Dia

Figura 9 - Variação horária do (a) saldo de radiação (Rn), (b) fluxo de calor no solo (G), (c) fluxo de calor latente (LE) e (d) fluxo de calor sensível (H) medidos e estimados nas áreas urbana e de cana-de-açúcar no município de Barra do Bugres-MT. 


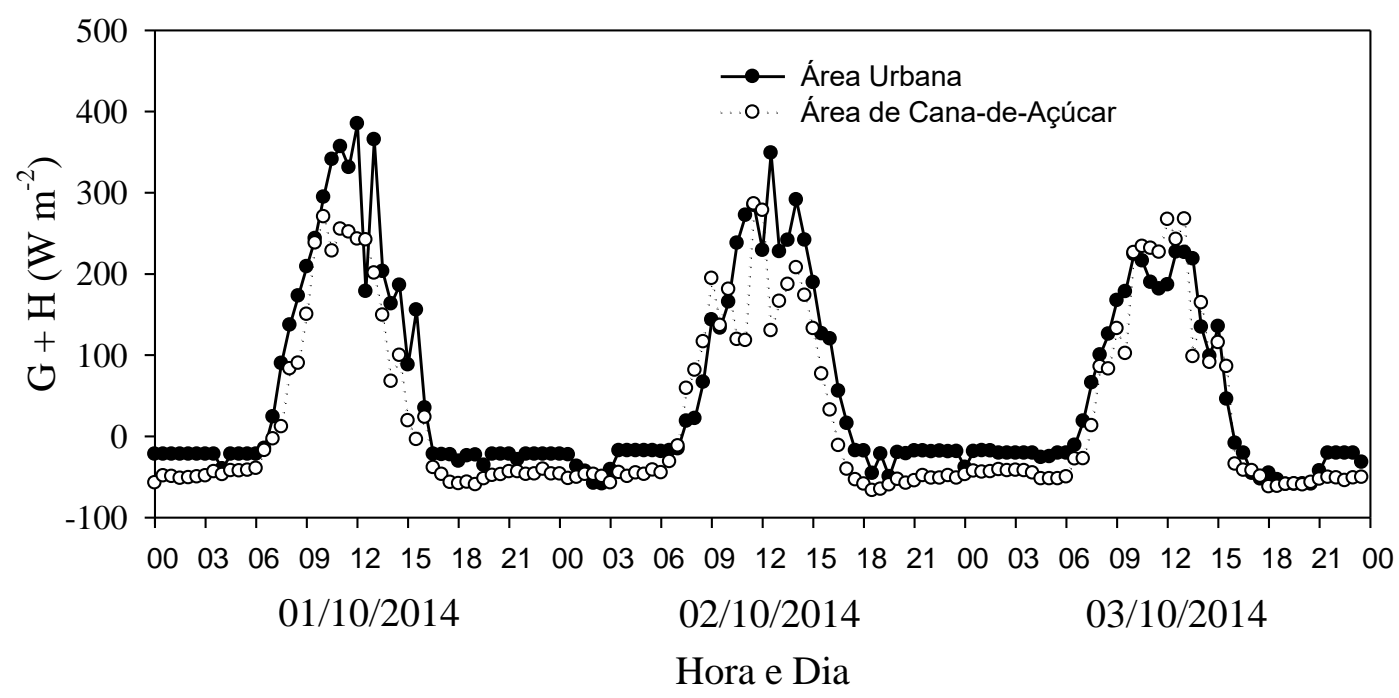

Figura 10 - Variação horária da soma do fluxo de calor no solo e do fluxo de calor sensível medidos e estimados na área urbana e de cana-de-açúcar no município de Barra do Bugres-MT.

\section{CONCLUSÃO}

Os sensores desenvolvidos apresentaram alta correlação quando comparados com os sensores de referência. A radiação solar medida nas duas estações micrometeorológicas tiveram padrão (amplitude e fase) semelhante, enquanto que, os padrões da temperatura e umidade relativa do ar e do balanço de energia foram diferentes nas duas áreas experimentais devido à diferente cobertura do solo.

Mais testes são necessários para avaliar a durabilidade dos sensores e datalogger desenvolvido. No entanto, as análises evidenciaram que o protótipo de estação micrometeorológica desenvolvido pode ser utilizado para estimar o balanço de energia.

\section{AGRADECIMENTOS}

A pesquisa foi apoiada pela Universidade do Estado de Mato Grosso (UNEMAT), Universidade Federal de Mato Grosso (UFMT), Programa de PósGraduação em Física Ambiental (PPGFA/IF/UFMT), Instituto Federal de Mato Grosso (IFMT), Conselho Nacional de Desenvolvimento Científico e Tecnológico (CNPq, processos no 310879/2017-5 e 305761/2018-8; Edital Universal 01/2016, processo no 407463/2016-0) e Fundação de Amparo à Pesquisa do Estado de Mato Grosso (FAPEMAT - PRONEM 2014, processo no 561397/2014).

\section{REFERÊNCIAS BIBLIOGRÁFICAS}

ALVES, E. D. L.; VECCHIA, F. A. S. Influência de diferentes superfícies na temperatura e no fluxo de energia: um ensaio experimental. Revista Ambiência, v. 8, n. 1. p. $101-111,2012$. 
ANDRADE, R. G.; SEDIYAMA, G. C.; DELGADO, R. C.; LIMA, E. P.; MENEZES, S. J. M. C. Saldo de radiação e fluxo de calor no solo na região do município de Juiz de Fora, MG. 1. (SERHIDRO). Anais I Seminário de Recursos Hídricos da Bacia Hidrográfica do Paraíba do Sul: o Eucalipto e o Ciclo Hidrológico. Taubaté: IPABHi. p. 371-373, 2007.

BILLESBACH, D. P.; ARKEBAUER, T. J. First long-term, direct measurements of evapotranspiration and surface water balance in the Nebraska SandHills. Agricultural and Forest Meteorology, v.156, p.104-110, 2012.

BIUDES, M. S.; VOURLITIS, G. L.; MACHADO, N. G.; ARRUDA, P. H. Z.; NEVES, G. A. R.; LOBO, F. A.; NEALE, C. M. U.; NOGUEIRA, J. S. Patterns of energy exchange for tropical eco systems across a climate gradient in Mato Grosso, Brazil. Agricultural and Forest Meteorology, v.202, p.112-124, March, 2015.

BIUDES, M.S.; CAMPELO JÚNIOR, J.H.; NOGUEIRA, J.S.; SANCHES. L. Estimativa do balanço de energia em cambarazal e pastagem no norte do Pantanal pelo método da razão de Bowen. Revista Brasileira de Meteorologia, 24(2), 135-143, 2009.

CALLEJAS, I. J. A. Avaliação temporal do balanço de energia em ambientes urbanos na cidade de Cuiabá-MT. 2012. 242f. Tese (Doutorado em Física Ambiental) - Universidade Federal de Mato Grosso 2012.

CALLEJAS, I. J. A.; NOGUEIRA, M. C. J. A.; BIUDES, M. S.; DURANTE, L. C. Seasonal variation of surface energy balance of a central Brazil city. Mercartor, v.15, n.3, p.85-106, 2016.

CHAKRABORTY, T; SARANGI, C.; TRIPATHI, S. N. Understanding Diurnality and Inter-Seasonality of a Sub-tropical Urban Heat Island. Boundary-Layer Meteorology, v.163, n.2, p.287-309, 2016.

CHRISTEN A.; VOGT, R. Energy and radiation balance of a central European city. International Journal of Climatology, v.24, p.1395-1421, 2004.

COUTTS , A. BERINGER, M.; J.; TAPPER, N. J. Impact of increasing urban density on local climate: spatial and temporal variations in the surface energy balance in Melbourne, Australia. Journal of Applied Meteorology and Climatology, v.46, p.477-493, 2007.

DICKEN, U.; COHEN, S.; TANNY, J. Examination of the Bowen ratio energy balance technique for evapotranspiration estimates in screenhouses. Biosystems Engeneering, v.114, p.397-405, 2013.

DREXLER, J.Z.; SNYDER, R.L.; SPANO, D.; PAW U, K.T. A review of models and micrometeorological methods used to estimate wetland evapotranspiration. Hydrological Processes, v.18, n.11, p.2071-2101, 2004.

FERREIRA, A. S; SIMÕES, M; MENDONÇA, J. C. Estimativas dos fluxos de energia em áreas com cultivo de cana-de-açúcar e soja na mesorregião do sul goiano. Anais XVI Simpósio Brasileiro de Sensoriamento Remoto - SBSR, Foz do Iguaçu, PR, Brasil, 13 a 18 de abril de 2013, INPE.

HU, S.; ZHAO, C.; LI, J.; WANG, F.; CHEN, Y. Discussion and reassessment of the method used for accepting or rejecting data observed by a Bowen ratio system. Hydrological Processes, v.28, n.15, p.4506-4510, 2013. 
IBGE, Instituto Brasileiro de Geografia e Estatística. Levantamento sistemático da produção agrícola: pesquisa mensal de previsão e acompanhamento das safras agrícolas no ano civil. Brasília, DF, 2014.

NEVES G. A. R. Desenvolvimento de Estação Micrometeorológica com Armazenamento de Dados. 61f. Dissertação (Mestrado em Física Ambiental). Universidade Federal de Mato Grosso, 2011.

NEVES, G. A. R. Desenvolvimento de sensores alternativos e sistema automatizado de armazenamento de dados para coleta de variáveis micrometeorológicas. 81f. Tese (Doutorado em Física Ambiental) - Instituto de Física, Universidade Federal de Mato Grosso, 2013.

NEVES, G. A. R.; MARQUES, J. B.; NOGUEIRA, J. S.; BIUDES, M. S.; ARRUDA, P. H. Z.; CURADO, L. F. A.; PALÁCIOS, R. S. Desenvolvimento e calibração de um termohigrômetro para uso em pesquisas de micrometeorologia, agrometeorologia e climatologia. Revista Brasileira de Geografia Física, v.8, n.1, p.136-143, 2015.

ORTEGA FARIAS, S. O.; CUENCA, R. H.; EK, M. Daytime variation of sensible heat flux estimated by the bulk aerodynamic method over a grass canopy. Agricultural and Forest Meteorology, v.81, p.131-143, 1996.

PEREZ, P. J.; CASTELLVI, F.; IBAÑEZ, M.; ROSELL, J. I. Assessment of reliability of Bowen ratio method for partitioning fluxes. Agricultural and Forest Meteorology, v.97, n.3, p.141-50, 1999.

QUERINO, C. A. S.; BIUDES, M. S.; MACHADO, N. G. M.; SANTOS NETO, L. A.; SILVA, M. J. G.; ARRUDA, P. NOGUEIRA, J. Balanço de ondas curtas sobre floresta sazonalmente alagável do Pantanal mato-grossense. Revista Brasileira de Climatologia, v.20, p. 252 - 266, JAN/JUN, 2017.

ROMISIO, G. B. A.; MENDONÇA, J. C.; PINHEIRO, F. M. A.; MARQUES, V. S.; JOSÉ M. Aspectos energéticos do desenvolvimento da cana-de-açúcar. Parte 2: balanço de energia e parâmetros derivados. Revista Brasileira de Meteorologia, v.25, n.4, p. 535 - 542, 2010.

SANO, E. E.; ROSA, R.; BRITO, J. L. S.; FERREIRA JR., L. G. Land cover mapping of the tropical savanna region in Brazil. Environmental Monitoring and Assessment, 66, p. 113-124, 2010.

SOUZA, D. M.; CARFAN, A. C.; NERY, J. T. Análise da temperatura do ar e de fluxos de calor no município de Ourinhos, utilizando o modelo envi-met. Revista Geonorte, Edição Especial, V.2, N.4, p.723 - 733, 2012.

UNLAND, H.E.; HOUSER, P.R.; SHUTTLEWORTH, W.J.; YANG, Z. Surface flux measurement and modeling at a semi-arid Sonoran Desert site. Agricultural and Forest Meteorology, v.82, p.119-153, 1996 